\title{
Altmetrics: métricas alternativas de impacto científico com base em redes sociais
}

\section{Moreno Barros}

Bibliotecário da UFRJ. Doutor em História das Ciências

http://dx.doi.org/10.1590/1981-5344/1782

Métricas alternativas ou "altmetrics" podem ser definidas como o estudo e uso de medidas de impacto acadêmico com base na atividade de ferramentas e ambientes online. Altmetrics visam medir as diferentes formas e padrões de significado e uso dos produtos científicos, verificando não apenas a publicação em veículos tradicionais, mas também o processo mais amplo de disseminação em ambientes emergentes. Seu objetivo é atualizar o conceito de influência científica em um século caracterizado pela rápida disseminação de informações e adoção de mídias sociais em escala global. O presente trabalho apresenta as altmetrics e analisa sua aplicação para avaliar o impacto acadêmico.

Palavras-chave: Almetrics; Webometria; Bibliometria; Fator de Impacto; Redes Sociais.

\section{Altmetrics: alternative metrics of scientific impact based on social media}

\begin{abstract}
Alternative metrics or "altmetrics" can be defined as the study and use of academic impact measures based on the activity of online tools and environments. Altmetrics aim to measure the different forms and patterns of meaning and use of scientific products, looking not only for publishing in traditional media, but also the broader process of dissemination in emerging environments. Its goal is to update the scientific concept of influence in a century characterized by the rapid spread of information and adoption of social media on a global scale. This paper presents the altmetrics and analyses its application to assess academic impact.
\end{abstract}


Keywords: Altmetrics; Webometrics; Bibliometrics; Impact Factor; Social Media.

Recebido em 21.12.2013 Aceito em 07.05.2015

\section{Introdução}

Cientometristas e gestores científicos há décadas rastreiam as menções formais para informar a medição, avaliação e estudo da ciência, graças ao fato de que as citações refletem 0 uso dos produtos acadêmicos. Mas apesar de serem úteis para a avaliação de pesquisadores e publicações, citações refletem apenas um segmento restrito do espectro do impacto científico, o artigo de periódico. Como Haustein (2012) apontou, esta abordagem da contagem de citações ignora vários outros aspectos que contribuem para a posição de um produto acadêmico. Só uma abordagem multidimensional seria capaz de analisar o impacto de um pesquisador ou periódico suficientemente.

Muito dos produtos científicos permanece sem citação e o Fator de Impacto de periódicos (Journal Citation Reports) naturalmente ignora isso. Mais importante, talvez, a cobertura do Fator de Impacto é limitada pelo tempo que leva para um artigo acumular citações. Alternativas métricas com base em citações, como o Eigenfactor e Índice h, corrigem algumas das falhas do Fator de Impacto, mas ainda sofrem com esse atraso.

$\mathrm{Na}$ etapa de avaliação da qualidade científica os pesquisadores se voltam a essas instâncias de reconhecimento pelos pares (MERTON, 1979) para ajudar a controlar o fluxo de ideias acadêmicas e são recompensados com um entendimento de como o conhecimento científico é transmitido e adotado. No entanto, o rastreamento da citação nunca foi capaz de acompanhar os tópicos menos visíveis, mas muitas vezes mais importantes dos colégios invisíveis, tecidos através de ligações pessoais e comunicações informais. Priem, Piwowar e Hemminger (2012) indicam que a atual reificação online destes tópicos etéreos nos dá a chance de preencher essa lacuna, construindo um mapa mais profundo e mais rico dos fluxos de informações científicas.

Como consequência, há um interesse crescente em um novo tipo de métrica com base na web que pode escalar de forma mais eficaz e apresentar um quadro ainda mais amplo do impacto científico: métricas baseadas em atividades de mídia social. O aumento do uso acadêmico de ferramentas web apresenta uma oportunidade para acompanhar os impactos alternativos, caminhos até então invisíveis, que estão começando a deixar vestígios online (PRIEM et al., 2012). Esses rastros se tornam visíveis em páginas da web, blogs, downloads, redes sociais como Twitter e Facebook, e gerenciadores de referências bibliográficas como CiteULike, Mendeley e Zotero.

Ferramentas de mídia social estão se tornando cada vez mais importantes nos fluxos de trabalho dos pesquisadores, como vários estudos recentes têm evidenciado (CORRÊAA, 2011; PINHEIRO, 2012; 
PESSONI, 2012). Um item de avaliação adotado recentemente pelo Conselho Nacional de Desenvolvimento Científico e Tecnológico (CNPq) refere-se às iniciativas de educação e divulgação científicas feitas pelos próprios cientistas. O CNPq avaliará no critério "divulgação" se os cientistas possuem blogs pessoais sobre ciência e se divulgam à mídia os resultados de seus trabalhos (CNPq, 2012). Se antes se valorizava apenas a produtividade acadêmica na avaliação de seu desempenho, hoje o pesquisador precisa se conscientizar da importância de fazer divulgação online de seus produtos de pesquisa.

É importante ressaltar que essas ferramentas não criam novos tipos de prática acadêmica tanto quanto facilitam práticas existentes. Gerenciadores sociais de referências, por exemplo, são uma extensão das coleções bibliográficas em papel que os pesquisadores têm mantido ao longo dos séculos, enquanto Twitter e Facebook facilitam o tipo de conversas informais que há muito tempo existem em diversos espaços acadêmicos. Cronin et al. (1998) reconhecem que essas ferramentas abrem uma janela valiosa sobre os até então escondidos processos de pesquisa, finalmente cumprindo a promessa do início da web de garantir substância a modos de influência que têm sido historicamente legados a segundo plano nas narrativas da ciência.

Encontrar formas de legitimamente alimentar o processo de acreditação acadêmica é uma necessidade constante para garantir as funções basilares de investimento e reconhecimento dentro da academia. Assim, neste artigo, apresentamos o uso das métricas baseadas na web, em particular aquelas que se apropriam das mídias sociais para avaliar o impacto de produtos científicos. A maturidade destas métricas deve abrir caminho para que sejam também reavaliadas as medições sobre a produtividade e autoridade dos pesquisadores. Enquanto alguns autores propõem uma espécie de revisão por pares leve, "altmetrics" tem sido utilizadas para descrever métricas alternativas baseadas em mídia social na web, com os proponentes divulgando sua velocidade, riqueza e amplitude.

\section{Métricas alternativas}

Pesquisas recentes no campo da webometria apresentam possibilidades interessantes para ir além das medidas tradicionais do impacto dos resultados das pesquisas, especialmente as citações na literatura acadêmica. Métricas alternativas e multidimensionais para a avaliação dos produtos científicos podem ser resumidas sob o conceito de "altmetrics" e refletem a visão de que é possível ampliar o escopo da revisão por pares fazendo uso de tecnologias colaborativas na web.

As chamadas métricas alternativas ou altmetrics podem ser definidas como o estudo e uso de medidas de impacto acadêmico com base na atividade de ferramentas e ambientes online, e nesse sentido ser encaradas como um subconjunto da webometria e da cientometria. 0 termo também tem sido utilizado para descrever as métricas propriamente (PRIEM; GROTH; TARABORELLI, 2012). 
Em razão da comunicação acadêmica ter migrado essencialmente para a web, usos anteriormente ocultos como a leitura, compartilhamento, discussões e classificações estão começando a deixar rastros online. Ferramentas web e outras medidas online presumivelmente indicam caminhos que os leitores percorreram influenciados por um artigo. McFedries (2012) explica que, em suma, qualquer "burburinho" o artigo está a gerar na web é um elemento potencial de medição altmétrica.

Altmetrics visa utilizar dados da web (ou seja, volume de tweets, curtidas, favoritos, comentários em blogs, etc.) e ferramentas da web (redes sociais, sistemas de bookmarking social ${ }^{1}$, gerenciadores de referências bibliográficas, etc) para compreender plenamente as características da comunicação científica na web. Além disso, altmetrics credita atividades acadêmicas ("linkar" ou discutir artigos de periódicos em blogs, por exemplo) realizadas na web que ainda não são reconhecidas por métricas tradicionais de impacto científico (como os indicadores de citação).

As altmetrics se baseiam em informações de uso em mídia social e podem ser empregadas lado-a-lado com as citações - um acompanhando a influência formal, reconhecida e o outro rastreando a certificação não intencional e informal. São capazes também de fornecer informações sobre o impacto em diversos públicos, especializado e leigo, bem como ajudar a controlar o uso de diversos produtos de pesquisa, tais como conjuntos de dados, softwares e blogs.

Altmetrics fazem parte do que os pesquisadores (PRIEM; HEMMINGER, 2010) chamam de Cientometria 2.0, onde as técnicas de mineração de dados são exercidas sobre grandes bases de dados de mídia social e outros depósitos online em busca de novos indicadores de impacto acadêmico. Certamente não substituirão as medidas tradicionais, como o fator de impacto. Seu objetivo é simplesmente atualizar o conceito de influência científica em um século caracterizado pela rápida disseminação de informações e mídias sociais em escala global.

\section{Propriedades das almetrics}

Altmetrics possuem a importante vantagem da velocidade, normalmente se acumulando em dias ou semanas, em vez dos anos que as citações exigem. Isto é particularmente útil na medida que o processo de pesquisa aumenta seu ritmo e os usuários de conteúdo científico precisam entender rapidamente seu impacto. Além de serem mais rápidas do que as medidas tradicionais de citação, altmetrics podem medir diferentes tipos de produtos acadêmicos, medir o impacto sobre públicos não-acadêmicos e, mais importante, oferecer suporte a uma compreensão mais ampla, mais matizada de impacto (CRONIN, 2001).

Altmetrics podem apoiar mapas da ciência mais transparentes, avaliações mais amplas e equitativas, e melhorias para o sistema de

\footnotetext{
${ }^{1}$ Serviço online que permite aos usuários adicionar, anotar, editar e compartilhar seus documentos, páginas da web e websites favoritos.
} 
revisão por pares. Em vez de esperar meses por duas opiniões, o impacto de um artigo pode ser avaliado por milhares de conversas e marcações em uma semana. No curto prazo, esse processo é suscetível de completar a tradicional avaliação pelos pares.

A velocidade de altmetrics apresenta a oportunidade de criar recomendação em tempo real e sistemas colaborativos de filtragem: em vez de assinar dezenas de alertas automáticos para os periódicos, um pesquisador poderia obter uma curadoria humana dos trabalhos mais significativos da semana corrente em seu campo. Isto torna-se especialmente poderoso quando combinado com rápidas publicações alternativas, como blogs, tweets ou servidores de pré-publicações, diminuindo o ciclo de comunicação de anos para semanas ou dias.

Além de traduzir as análises estatísticas para novas mídias e ferramentas, altmetrics tem a intenção de fazer uso de dados quantitativos e qualitativos (como marcadores ou hashtags geradas pelos usuários) para holisticamente avaliar o artigo, o impacto do periódico ou do autor, onde a influência não implica apenas ser citado, mas também ser lido, comentado, "tuitado", curtido e assim por diante (HAUSTEIN; PETERS, 2012).

Ao contrário de métricas de citação, altmetrics rastreia impacto fora da academia, a dispersão do trabalho influente, mas que não foi citado, e o impacto a partir de fontes que não passam pelo processo de revisão por pares tradicional. Alguns pesquisadores (SMEYERS; BURBULES, 2011) podem sugerir que as altmetrics seriam muito fáceis de burlar, mas não menos do que o próprio Fator de Impacto. Priem et al. (2010) acreditam que sistemas de altmetrics deverão atingir maturidade e ser mais robustos, aproveitando a diversidade de metrias alternativas e poder estatístico da big data para algoritmicamente detectar e corrigir atividade fraudulenta. Esta abordagem já funciona para os anunciantes online, sites de notícias sociais, Wikipedia e motores de busca.

Como resultado, altmetrics visam medir as diferentes formas de padrões de significado e uso, verificando não apenas a publicação final, mas também o processo mais amplo de pesquisa, colaboração e contatos em torno das publicações de pesquisa. O futuro, então, poderia enxergar altmetrics e bibliometria tradicional apresentadas em conjunto como ferramentas complementares, apresentando uma visão diferenciada, multidimensional de pesquisas de impacto em diferentes escalas de tempo.

\section{Propósito das altmetrics}

As universidades enfrentam crescente pressão devido ao financiamento reduzido acompanhado de expectativas de retorno sobre o investimento, que terminam por exigir acesso mais amplo e mais rápido aos resultados de pesquisa. A mais significativa dessas pressões é o incremento da ênfase no número de publicações dos pesquisadores, como medida para classificações de programas de pesquisa e da instituição como um todo. Tanto pesquisadores como financiadores realizam 
julgamentos rotineiros sobre a melhor forma de investir seus limitados tempo e dinheiro, o que se torna cada vez mais difícil em função do volume da comunicação científica.

Como a literatura científica cresceu enormemente, financiadores e avaliadores dependem de filtros para selecionar os trabalhos mais relevantes e significativos. Em meados do século 20, filtros construídos sobre uma base de indexação manual começaram a quebrar sob o peso da produção da ciência pós-guerra em franca expansão. Como solução, filtros automatizados foram propostos para alavancar os juízos de impacto dos cientistas, agregando a incidência de citações como indicação de reconhecimento pelos pares. Estes filtros baseados em citações cresceram dramaticamente em importância e tornaram-se o princípio de como o impacto da pesquisa é medido.

Quando se trata de avaliar a qualidade do trabalho de um pesquisador para promoção ou outros fins, uma medida de referência para a qualidade da pesquisa é frequentemente utilizada. O número de vezes que uma obra em particular foi citada tem sido tradicionalmente utilizado para medir a qualidade da produção de um pesquisador em relação a outros em um mesmo campo. Os avaliadores muitas vezes dependem, em particular, do Fator de Impacto de Periódicos da Thomson Scientific, no entanto, a taxa de citação de um artigo não é mais a única métrica disponível para avaliar o impacto acadêmico. Como a indexação manual, há 60 anos, avaliadores e financiadores podem hoje estar fracassando em se manter em dia com o crescimento do volume literatura, sua velocidade e diversidade.

Desde o final do século 20, no entanto, as agências de financiamento começaram a solicitar que os proponentes indiquem, e revisores avaliem, não só o mérito intelectual das atividades propostas, mas também os seus potenciais impactos sociais mais amplos. Acadêmicos podem ser julgados de acordo com a capacidade de outros pesquisadores construirem ou reutilizarem os resultados de suas pesquisas originais.

Os artigos científicos tradicionais estão cada vez mais acompanhados de conjuntos de dados, códigos e projetos experimentais, publicação em menor escala onde a unidade citável é um argumento ou passagem em vez do artigo inteiro, auto-publicação generalizada via blogs, microblogging e comentários ou anotações sobre trabalhos existentes. Conforme estes diferentes tipos de produtos acadêmicos se tornam importantes para futuras pesquisas, encontrar formas para que se incorporem ao processo de acreditação acadêmica é necessário para garantir as funções essenciais de investimento e reconhecimento dentro da academia.

Ademais, pesquisadores têm pouco incentivo para experimentar novas formas de comunicação científica ou tornar suas pesquisas disponíveis livremente na velocidade da ciência, uma vez que são reconhecidos principalmente pela publicação de artigos de periódicos e livros. Por muito tempo, a publicação acadêmica tem contado com uma infraestrutura auto-referenciada que foi projetada para atender a 
interesses específicos. Pesquisadores que tentam praticar ciência em modo aberto podem achar difícil obter o reconhecimento para o impacto de publicações e produtos de pesquisa além do artigo de periódico, como produtos que incluem software científico, dados, e assim por diante.

Devemos considerar se altmetrics podem servir aos proponentes e revisores para enfrentar o que se tornou uma questão crítica na avaliação das propostas de concessão de financiamento, cargos e salários, que é analisar os potenciais impactos mais amplos das atividades propostas. Altmetrics podem representar uma camada de avaliação adicional, onde mesmo em situações em que a pesquisa poderia ser melhor servida por uma publicação de um conjunto de dados, publicação de blog ou outro produto de pesquisa com base na web, a carreira do pesquisador é reduzida à aplicação de esforço na confecção de um artigo tradicional. Em vez de avaliar apenas os artigos criados em papel, livros e anais, altmetrics sugerem a construção de um novo sistema em que todos os tipos de produtos acadêmicos são avaliados e recompensados.

Além disso, o trabalho de comunicação científica e impacto dos artigos publicados são principalmente dependentes da visibilidade dos autores e suas publicações, uma vez que só a consciência da existência dos artigos induz leitores, e junto com ele citações. Sobretudo, mídias sociais e novas ferramentas para a divulgação de informação e comunicação podem auxiliar não só avaliadores, mas os próprios pesquisadores a abordar novos públicos, publicar os resultados de pesquisa, ou orientar os leitores para publicações relevantes, afirmando o valor da democratização dos espaços para a comunicação científica.

\section{Almetrics em ação}

Para os propósitos deste artigo, considerando a fase prematura que se encontra o conceito de métricas alternativas, é conveniente apresentar algumas das ferramentas de altmetrics mais importantes dentro do ecossistema de publicação acadêmica, a fim de ilustrar como se dá a aplicação dessas ferramentas na prática. A seguir, procuramos descrever brevemente algumas plataformas altmetrics e explicar como estas comunicam as métricas de pesquisa visando proporcionar uma melhor compreensão do impacto acadêmico.

a) Altmetric (altmetric.com)

Altmetric oferece como produto principal o programa Explorer, que fornece informações sobre quantas vezes um artigo foi visualizado juntamente do ranking do periódico em que foi publicado (similar aos indicadores oferecidos pelo Scielo, por exemplo). Mas além disso, Explorer oferece uma lista de componentes sociais, como quantas vezes um artigo foi visitado a partir de um feed de notícias, quantas vezes ele foi "tuitado" ou "retuitado", quantas "curtidas" e discussões gerou no Facebook e no Google +, bem como diversas outras plataformas de mídia social. Usando Explorer, um pesquisador pode visualizar a demografia de quem acessou seu artigo. 
Cada semana o Altmetric captura de centenas de milhares de tweets, posts, notícias e outros vestígios de conteúdo que mencionam artigos acadêmicos. O Explorer é capaz de navegar, pesquisar e filtrar esses dados, recolhendo as discussões pertinentes ao redor de cada artigo no Twitter, Facebook, blogs de ciência, meios de comunicação tradicionais e outras fontes mais, para então tornar tudo disponível em um único painel de gerenciamento.

Figura 1 - Painel Altmetric para as publicações da PLoS, oferecendo visualização de todos os artigos mencionados em mídias sociais no último mês. O número indicado no círculo representa uma contabilização de menções no Twitter, Facebook, Google + e outras fontes de mídia e notícias

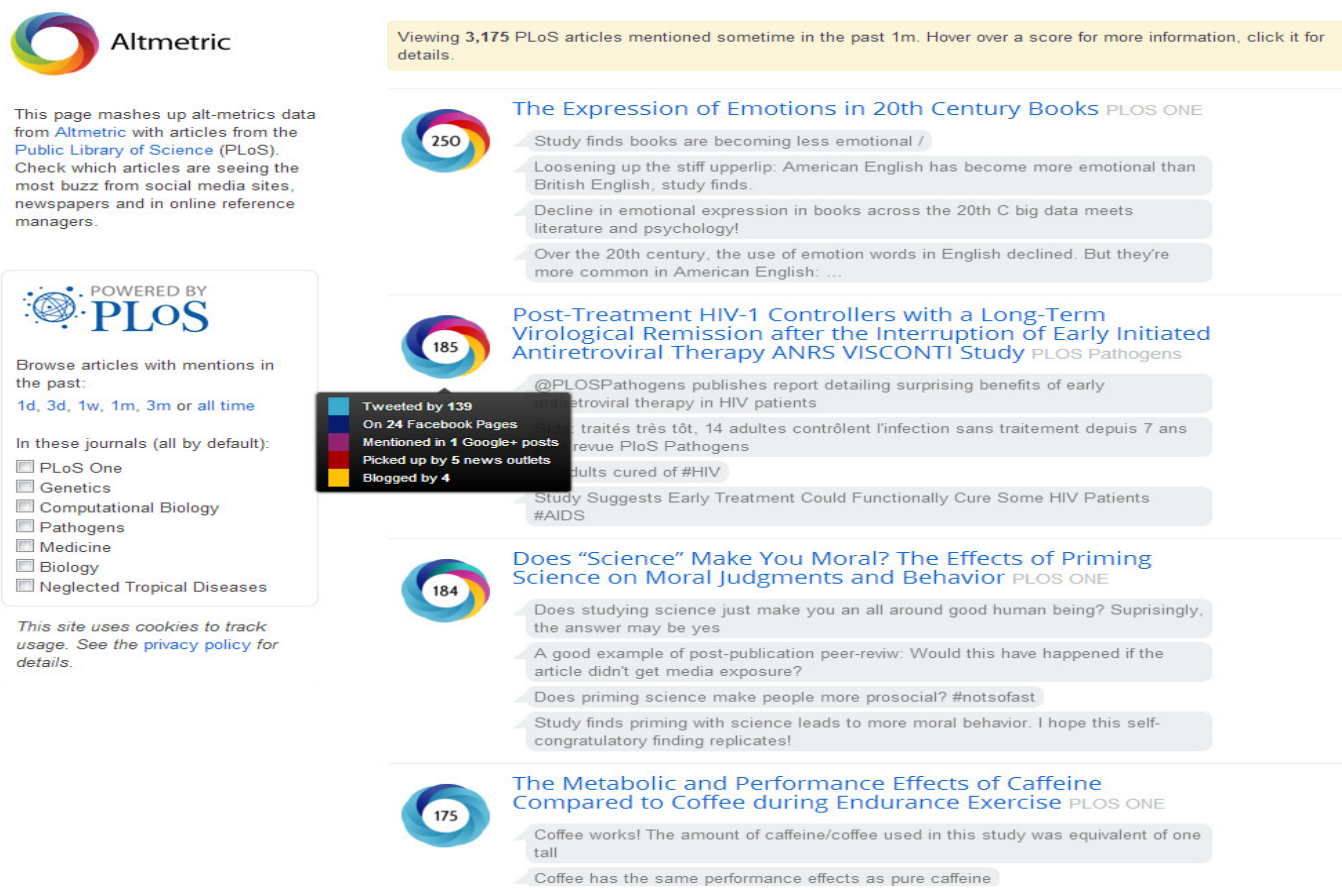

Fonte: print screen da aplicação Altmetric. Disponível em:

<http://altmetric.com/demos/plos.html>. Acesso em: 26 set. 2012.

b)Impact Story (impactstory.org)

ImpactStory rastreia métricas através de uma variedade de serviços comumente utilizados, tais como Delicious, Scopus, Mendeley, PubMed, SlideShare, entre outros. O pesquisador pode importar seu perfil do Google Scholar ou os registros DOI de seus artigos. Depois de importar o material que deseja medir, o Impact Story informa quantas vezes um artigo foi salvo pelos leitores, quantas vezes foi citado por acadêmicos, quantas pessoas o têm discutido publicamente via Twitter, Facebook, etc e quantas vezes ele foi citado pelo grande público (artigos da Wikipedia, posts de blogs, por exemplo).

Qualquer pessoa que tenha material de pesquisa em uma das plataformas que suporta o ImpactStory pode ver suas métricas individualmente, criando sua própria coleção. Pesquisadores podem incorporar um widget em seus sites que irá anexar métricas do 
ImpactStory para suas citações, indicando se um artigo é altamente discutido ou citados por acadêmicos e pelo público.

FigURA 2 - Painel ImpactStory mostrando as métricas compiladas de visualizações acadêmicas e pelo público em geral, referências salvas no CiteULike e no Delicious e citações na Scopus

ImpactStory.

Moreno Barros embed follow about 1 products (expand all)

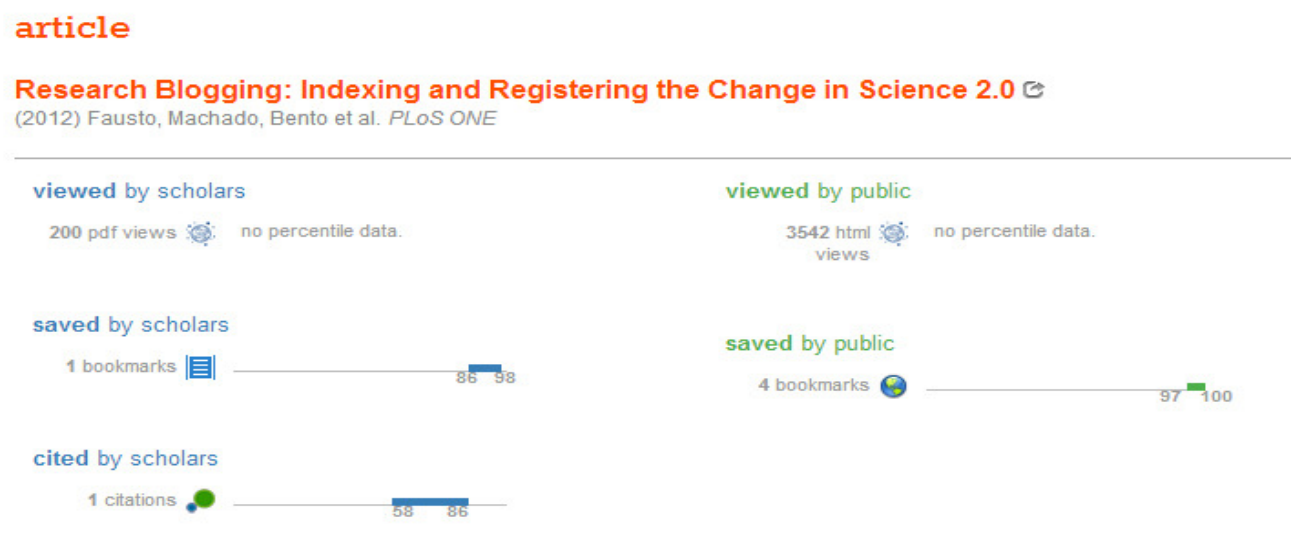

Fonte: Artigo da amostra: FAUSTO, S. et al. Research blogging: indexing and registering the change in Science 2.0. PloS one, v. 7, n. 12, 2012.

DOI: $10.1371 /$ journal.pone.0050109.

c)Nature Communications Metrics

Métricas ao nível do artigo estão disponíveis em 20 periódicos da plataforma Nature Publishing Group. Usuários da Nature.com podem visualizar os dados de citação, visualizações, menções na grande mídia, posts e compartilhamentos sociais, incluindo Facebook e Twitter, de qualquer artigo. Esta funcionalidade está disponível em todos os artigos de pesquisa publicados a partir de 2011.

Dados de citação são extraídos da Web of Science, Scopus e CrossRef e são atualizados diariamente. A contagem cumulativa de visualiazões para um artigo no site da Nature pode ser obtida por meio de um gráfico interativo. Notícias, posts, dados do Google+, Facebook, Twitter e Reddit são fornecidas pela ferramenta Altmetric por meio de uma interface de programação de aplicativo (API). 
Figura 3 - Visualização das métricas ao nível do artigo na publicação Nature Communications

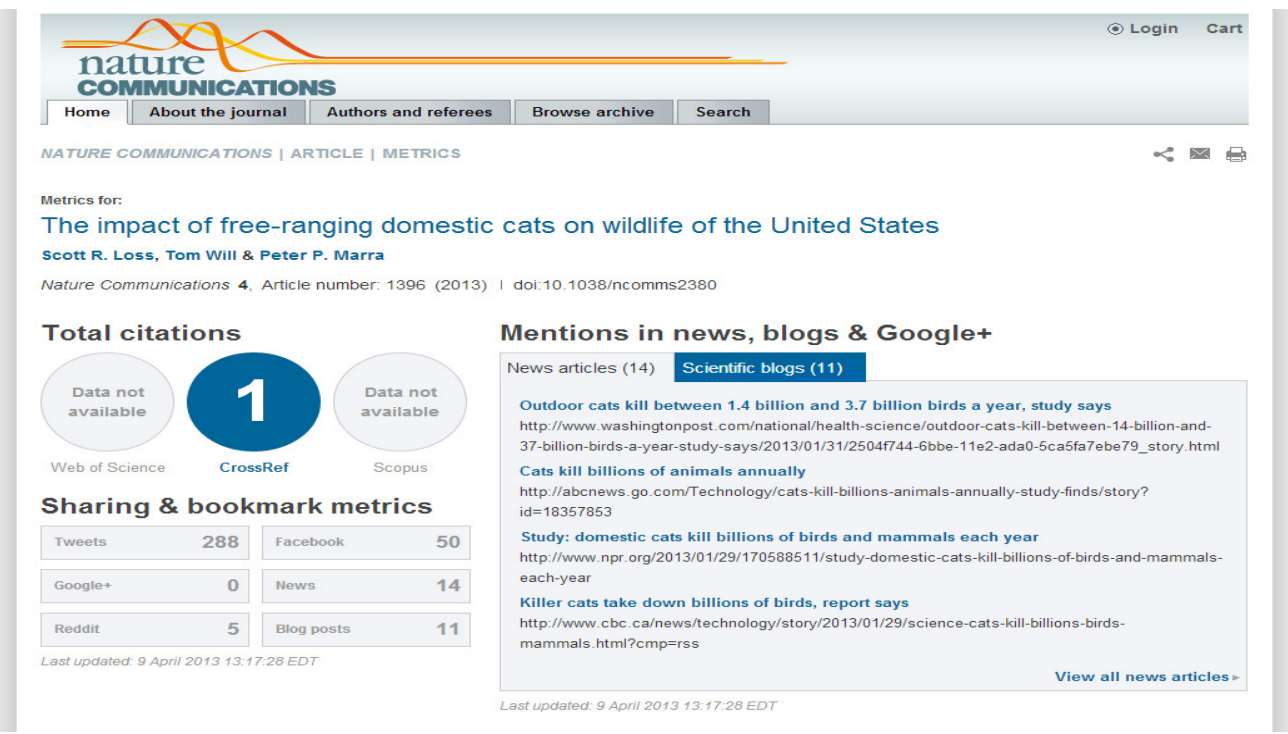

Fonte: print screen da publicação Nature Communications. Disponível em:

<http://www.nature.com/ncomms/journal/v4/n1/ncomms2380/metrics >. Acesso em: 26 set. 2012.

\section{d)PloS Article Level Metrics}

Métricas ao nível do artigo estão disponíveis para cada artigo publicado pela PloS (Public Library of Science). Essas métricas são um conjunto abrangente de indicadores de impacto, incluindo: uso; citações; bookmarking social e atividade de disseminação; cobertura na mídia e em blogs; atividades de discussão e classificações.

Figura 4 - Métricas ao nível do artigo na PloS.

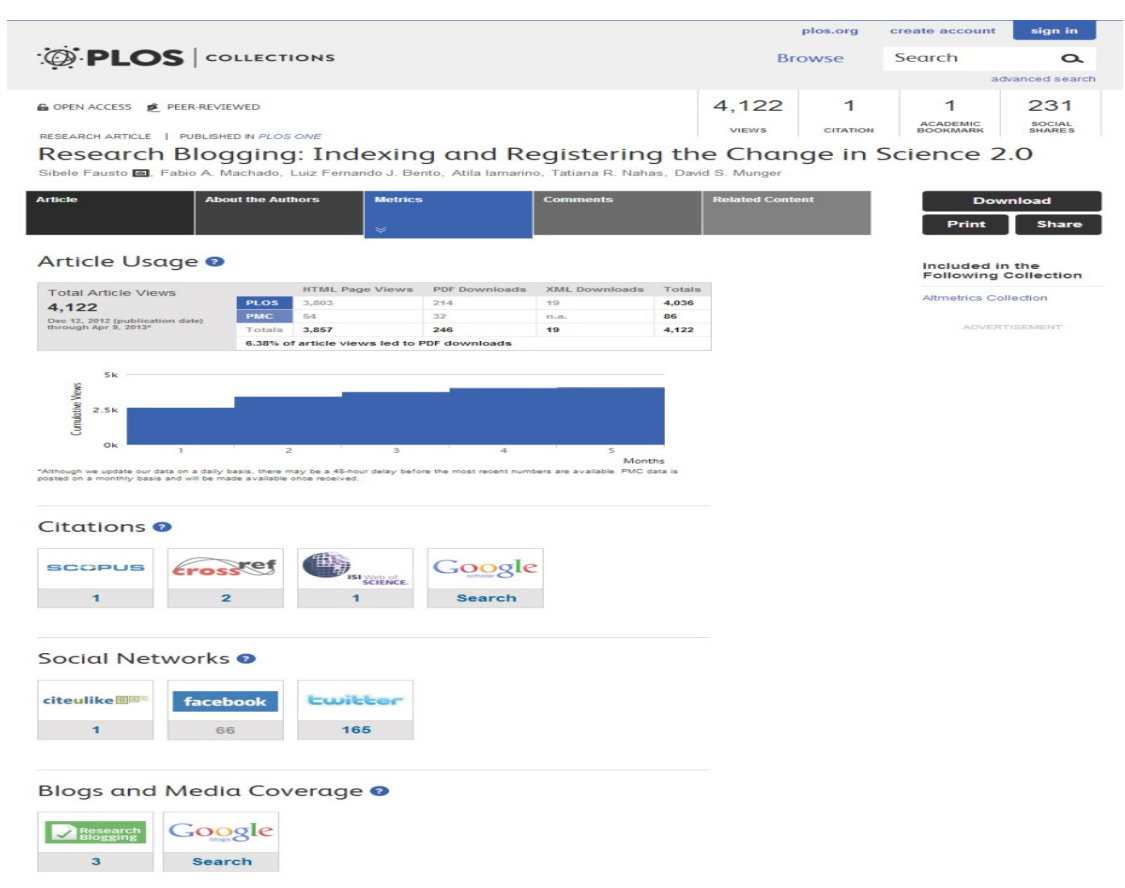

Fonte: FAUSTO, S. et al. Research blogging: indexing and registering the change in Science 2.0. PloS one, v. 7, n. 12, 2012. DOI:10.1371/journal.pone.0050109. 
Figura 5 - Métricas ao nível do artigo na PloS.
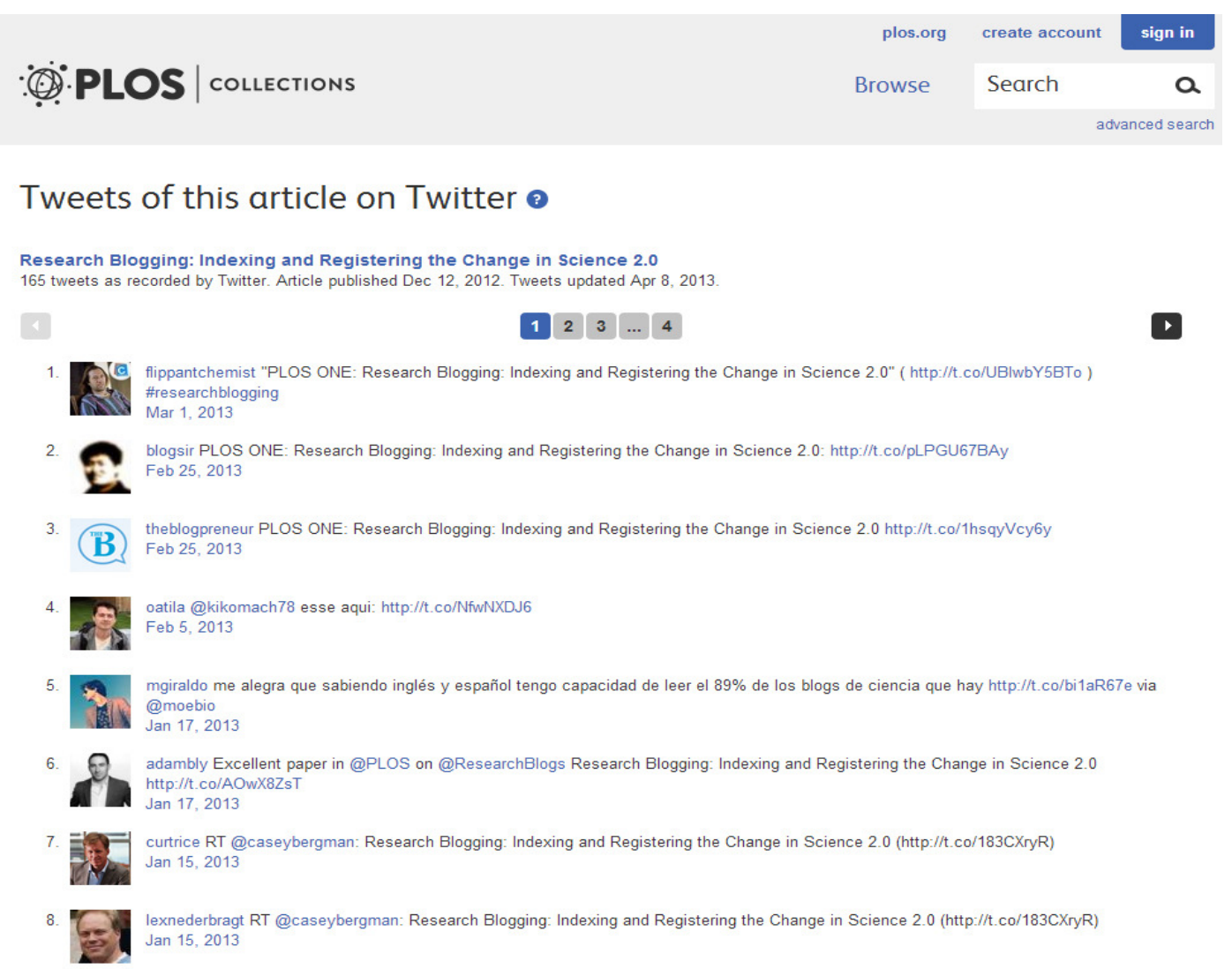

Fonte: Menções no Twitter para o artigo: FAUSTO, S. et al. Research blogging: indexing and registering the change in Science 2.0. PloS one, v. 7, n. 12, 2012.

DOI: $10.1371 /$ journal.pone.0050109.

Outras ferramentas adequadas para medição de altmetrics começam a proliferar e necessitam de melhor avaliação, entre elas Plum Analytics (plumanalytics.com), ScienceCard (sciencecard.org), PaperCritic (papercritic.com). É mister ressaltar que essas ferramentas só farão sentido no cenário nacional se os pesquisadores oferecerem dados passíveis de rastreamento que permitirão uma abordagem de impacto científico multidimensional, para além da tradicional publicação em periódicos Qualis e citações formais.

\section{Presença online}

A mudança de comportamentos de pesquisa para a web em vários domínios expõe mais e mais processos diversos de produção do conhecimento e comunicação científica. O comportamento online é rastreável $e$, consequentemente, a presença dos cientistas na web fornecem um conjunto adicional de rastros de informação para estudar a ciência, terreno bastante fértil para a aplicação das altmetrics.

É notório que com o aumento geral do uso da Internet, uma porção crescente de trabalho dos pesquisadores passou a ocorrer online, através de trocas de e-mail, acesso a bases de dados bibliográficas online, blogs, colaborando através de ferramentas de ciência eletrônica, bem como o 
uso da web em geral (BIRKHOLZ et al., 2012). Entretanto, estudos anteriores de Bar-Ilan et al. (2012) sobre a visibilidade dos cientistas na web indica que o impacto do autor sobre não-autores, isto é, leitores puros ou leigos, é perdido em contagens de citações tradicionais.

Embora a bibliometria e cientometria não tenham como foco específico redes sociais online como Twitter e Facebook, o campo está cada vez mais envolvido neste tipo de medição da atividade científica e do comportamento dos pesquisadores nesses ambientes. Diante da crescente popularidade das mídias sociais e considerando o compartilhamento de informação um componente central do trabalho de pesquisa, Cronin (2001) apela a uma maior investigação sobre os vários tipos de invocações baseados na web, o que promove uma imagem mais apurada da presença e influência online dos pesquisadores.

Antes que isso possa ser feito, no entanto, é importante descrever e caracterizar ferramentas de mídia social como fontes métricas. Usuários acadêmicos de ferramentas como o Twitter e Mendeley e continuam a ser minoria entre vezes seus pares. A fim de tornar as métricas alternativas uma realidade, alem da infraestrutura já disponível, deve haver uma cultura aberta de compartilhamento de informações. Assim como houve oposição por segmentos da comunidade acadêmica para 0 estabelecimento de normas para a publicação online no final da década de 1990, existe hoje oposição ao fornecimento de dados no nível mais granular necessária para métricas alternativas.

A baixa presença e atividades observadas em múltiplos websites indica que os pesquisadores da área de Ciência da Informação no Brasil, particularmente, não estão utilizando plataformas web como extensão de seu trabalho, inibindo a adoção plena de altmetrics e abrindo mão de proporcionar um apoio adicional na utilização de plataformas que facilitam a produção e comunicação de conhecimento.

O próprio exame de sistemas de comentários associados aos periódicos brasileiros de Ciência da Informação revela uma distribuição idêntica: nenhuma das revistas incluídas em amostra (periódicos em Ciência da Informação classificados entre A1 e C no Sistema WebQualis da CAPES) oferece a possibilidade de comentários sobre os artigos, sistemas tão comuns em blogs e nas redes sociais, e primordial para a manutenção de uma rede social online, baseada em conversação e compartilhamento.

Com o objetivo de oferecer uma visão sobre os rastros deixados por pesquisadores da área da Ciência da Informação, e tomando como base uma lista compilada por Murakami (2011) sobre redes de citações dos periódicos da área de Ciência da Informação com classificação acima de B2 no WebQualis e publicados em 2011, investigamos a presença online de 25 autores da área, brasileiros ou que atuam no território nacional, dentre aqueles com maior número de interconexões (nós e hubs) entre si.

O método para seleção dos nomes dos pesquisadores apenas reflete os dados tabulados no trabalho supracitado. A metodologia desta breve análise também implica que não procurávamos em bases de dados bibliográficas online para a evidência de publicações ou suas páginas institucionais acadêmicas. Em vez disso, buscamos isolar a existência de 
atividade online na web social, incluindo blogs, microblogging e atividade em plataformas sociais.

O quadro abaixo indica com um simples sim (S) ou não (N) se vestígios e rastros puderam ser encontrados, associados aos produtos científicos e presença online dos pesquisadores investigados. Foram coletadas manualmente informações básicas disponíveis livremente na web sobre os pesquisadores, incluindo menções de seus produtos científicos em blogs pessoais ou institucionais, a existência de contas cadastradas e perfis em redes sociais como Twitter (twitter.com), Facebook (facebook.com) e LinkedIn (linkedin.com), apresentações realizadas pelo ou sobre o autor no SlideShare (slideshare.com) e adesão ao Google Scholar Citations (scholar.google.com/citations). Os resultados foram verificados de acordo com detalhes oferecidos pelos próprios pesquisadores, tais como a profissão, nome, filiação institucional e publicações. Informações imprecisas e ambíguas não foram incluídas.

Quadro 1 - Vestígios online dos pesquisadores brasileiros em Ciência da Informação

\begin{tabular}{|c|c|c|c|c|c|c|}
\hline Autor & Blog & Twitter & Facebook & Scholar & LinkedIn & SlideShare \\
\hline BARBOSA, Ricardo Rodrigues & $\mathrm{N}$ & $\mathrm{N}$ & $\mathrm{S}$ & $\mathrm{N}$ & $\mathrm{N}$ & $\mathrm{N}$ \\
\hline BARRETO, Aldo & $\mathrm{S}$ & $\mathrm{S}$ & $\mathrm{S}$ & $\mathrm{S}$ & S & $\mathrm{S}$ \\
\hline BUFREM, Leilah Santiago & $\mathrm{N}$ & $\mathrm{N}$ & $\mathrm{S}$ & $\mathrm{N}$ & $\mathrm{N}$ & $\mathrm{S}$ \\
\hline DANTAS, Marcos & S & $\mathrm{S}$ & $\mathrm{N}$ & $\mathrm{N}$ & $\mathrm{S}$ & S \\
\hline DIAS, Eduardo Wense & $\mathrm{N}$ & $\mathrm{N}$ & $\mathrm{N}$ & $\mathrm{N}$ & $\mathrm{N}$ & $\mathrm{S}$ \\
\hline DODEBEI, Vera & $\mathrm{N}$ & $\mathrm{S}$ & S & $\mathrm{S}$ & S & S \\
\hline GODOY VIEIRA, Angel Freddy & $\mathrm{N}$ & $\mathrm{N}$ & $\mathrm{N}$ & $\mathrm{N}$ & $\mathrm{N}$ & $\mathrm{N}$ \\
\hline GONZÁLEZ DE GÓMEZ, Maria Nélida & $\mathrm{N}$ & $\mathrm{N}$ & $\mathrm{N}$ & $\mathrm{N}$ & $\mathrm{N}$ & $\mathrm{N}$ \\
\hline GUIMARÃES, José Augusto Chaves & $\mathrm{N}$ & $\mathrm{N}$ & $\mathrm{N}$ & $\mathrm{N}$ & $\mathrm{N}$ & $\mathrm{N}$ \\
\hline LARA, Marilda Lopes Ginez de & $\mathrm{N}$ & $\mathrm{N}$ & $\mathrm{S}$ & $\mathrm{N}$ & S & $\mathrm{N}$ \\
\hline MILANI, Suellen Oliveira & $\mathrm{N}$ & $\mathrm{N}$ & $\mathrm{N}$ & $\mathrm{N}$ & $\mathrm{N}$ & $\mathrm{N}$ \\
\hline MOSTAFA, Solange Puntel & $\mathrm{N}$ & $\mathrm{N}$ & S & $\mathrm{N}$ & S & S \\
\hline Müller-Granzotto, Marcos José & $\mathrm{N}$ & $\mathrm{N}$ & $\mathrm{N}$ & $\mathrm{N}$ & $\mathrm{N}$ & $\mathrm{N}$ \\
\hline NEHMY, Rosa Maria Quadros & $\mathrm{N}$ & $\mathrm{N}$ & $\mathrm{N}$ & $\mathrm{N}$ & $\mathrm{N}$ & $\mathrm{N}$ \\
\hline ORLANDI, Eni de Lourdes Puccinelli & $\mathrm{N}$ & $\mathrm{N}$ & $\mathrm{N}$ & $\mathrm{N}$ & $\mathrm{N}$ & $\mathrm{N}$ \\
\hline PENTEADO, Cláudio Luis de Camargo & $\mathrm{N}$ & $\mathrm{N}$ & $\mathrm{N}$ & $\mathrm{N}$ & $\mathrm{N}$ & $\mathrm{N}$ \\
\hline PINHEIRO, Lena Vânia Ribeiro & $\mathrm{N}$ & $\mathrm{N}$ & $\mathrm{N}$ & $\mathrm{N}$ & $\mathrm{N}$ & $\mathrm{N}$ \\
\hline RENDÓN ROJAS, Miguel A. R. & $\mathrm{N}$ & $\mathrm{N}$ & $\mathrm{N}$ & $\mathrm{N}$ & $\mathrm{N}$ & $\mathrm{N}$ \\
\hline ROMÃO, Lucilia Maria Sousa & $\mathrm{N}$ & $\mathrm{N}$ & S & S & $\mathrm{N}$ & $\mathrm{N}$ \\
\hline SILVA, Jonathan Raphael Bertassi da & $\mathrm{N}$ & $\mathrm{N}$ & $\mathrm{N}$ & $\mathrm{N}$ & $\mathrm{N}$ & $\mathrm{N}$ \\
\hline SMIT, Johanna Wilhelmina & $\mathrm{N}$ & $\mathrm{N}$ & $\mathrm{S}$ & S & S & S \\
\hline TÁLAMO, Maria de Fátima G. Moreira & $\mathrm{N}$ & $\mathrm{N}$ & $\mathrm{N}$ & $\mathrm{N}$ & $\mathrm{N}$ & $\mathrm{N}$ \\
\hline TOGNOLI, Natália Bolfarini & $\mathrm{N}$ & $\mathrm{N}$ & S & $\mathrm{N}$ & $\mathrm{N}$ & $\mathrm{N}$ \\
\hline TOUTAIN, Lidia M. B. Brandão & $\mathrm{N}$ & $\mathrm{N}$ & $\mathrm{N}$ & $\mathrm{N}$ & $\mathrm{N}$ & $\mathrm{N}$ \\
\hline VALENTIM, Marta Ligia Pomim & $\mathrm{N}$ & $\mathrm{N}$ & S & $\mathrm{S}$ & $\mathrm{N}$ & S \\
\hline
\end{tabular}

Fonte: Dados da pesquisa.

Usuários acadêmicos de ferramentas como o Twitter e Facebook, em especial na área de Ciência da Informação no Brasil, continuam a ser uma minoria entre os seus pares. Ainda que o uso dessas ferramentas para fins de disseminação científica seja modesto, ele pode revelar padrões de uso interessantes. Percebemos uma espécie de efeito Mateus entre os pesquisadores analisados, que tendem a utilizar mais de uma mídia social após explorar a primeira e acabam se beneficiando em termos de 
visibilidade, em comparação aos que não utilizam. O Facebook assume a posição de rede social mais adotada, agregando pesquisadores em torno de interesses comuns e conexões individuais, diminuindo a tensão entre a presença online estritamente pessoal e a profissional.

O aumento do uso dos cientistas sobre a web como uma plataforma de compartilhamento de conhecimento apresenta uma série de questões metodológicas de validade e confiabilidade (BIRKHOLZ et al., 2012). Os indícios de baixa adoção e penetração em mídias sociais pelos pesquisadores brasileiros não precisam ser encarados com ceticismo conquanto algumas questões permaneçam sem respostas válidas. Priem, Piwowar e Hemminger (2012) questionam se há de fato dados disponíveis suficientes para a construção de métricas alternativas significativas. Se sim, como os dados são distribuídos através de ferramentas, usuários e do tempo? Os pesquisadores devem perguntar se altmetrics realmente refletem o impacto, ou apenas o burburinho vazio.

Um crescente corpo de pesquisa começou a testar as afirmações sobre o valor dos altmetrics, examinando duas questões principais: primeiro, quantos dados altmetrics existem e como eles são distribuídos e, segundo, o que as altmetrics medem e como correlacionam-se com a citação. Certamente há uma necessidade de pesquisa exploratória mais ampla para responder a estas e outras perguntas, apresentando descobertas descritivas preliminares para orientar investigações mais profundas.

Apesar de uma estimativa baixa de pesquisadores que estão ativamente usando ferramentas de mídia social para fins acadêmicos, esse número está crescendo consistentemente, no Brasil e e no exterior. É provável que em menos de uma geração, os pesquisadores da área nativos digitais revertam o quadro de ausência online por completo sem a necessidade de um incentivo acadêmico legado arbitrariamente de cima para baixo.

\section{Desafios às altmetrics}

Duas impressões despontam em nossa análise sobre a perspectiva de adoção das altmetrics por parte da comunidade acadêmica. A primeira é que existem muitos produtos diferentes de mídias sociais que podem servir de motriz para as altmetrics. A segunda é que a diversidade desses produtos não é consistentemente utilizada pelos pesquisadores. No quesito técnico, existem diferentes ferramentas de mídia social, muitas das quais realizam processos semelhantes e não é tarefa fácil apresentar dados de todas elas em um único painel agregador.

Em relação ao comportamento dos acadêmicos, Gosling (2013) sugere que compartilhar seu trabalho de pesquisa com uma audiência mais ampla parece uma boa ideia, mas se esse ato compromete a possibilidade de publicar os resultados originais do trabalho em periódicos prestigiados em sua área, o pesquisador não terá a motivação para fazêlo. 
Enquanto os artigos de periódicos continuarem a ser vistos como o produto de pesquisa padrão e serem utilizados para julgar processos admissionais, promoções e concessões de subvenções e bolsas, existe o risco de não haver adoção em larga escala desses tipos de ferramentas para publicar resultados de pesquisa. Além disso, os pesquisadores podem argumentar que não dispõem do tempo necessário para divulgar em ambos lugares, de modo que o que conta para a sua carreira no momento é o que irão priorizar.

Barr (2013) entende que os julgamentos de pares prestados via crowdsourcing $^{2}$ na web aceleraria o processo de revisão e tornaria as revisões visíveis e acessíveis, mas as sentenças não estariam imunes à promoção da convencionalidade, isto é, o pensamento do grupo ou exclusividade cultural em maior escala, nem limitaria o volume de pesquisas publicadas. Embora o emprego de altmetrics para filtrar pesquisas impactantes ou significativas representasse um pouco a democratização da avaliação de pesquisas publicadas, ele também centralizaria e concentraria as decisões relativas à direção de trajetórias de pesquisa para as mãos de quem projeta e administra as métricas apenas uma subseção da comunidade acadêmica.

Medidas novas e complexas podem distorcer a imagem do sistema de ciência, se não forem rigorosamente avaliadas e corretamente entendidas. Obviamente, o uso e desenvolvimento de altmetrics deve ser prosseguido com cautela científica adequada. Priem, Groth e Taraborelli (2012) alertam que as altmetrics podem enfrentar tentativas de manipulação semelhante ao que o Google lida com os ranking de resultados de busca na web. Enfrentar tal manipulação pode, em sua vez, afetar a transparência das altmetrics. Uma nova frente de trabalho deve correlacionar altmetrics e as medidas existentes, prever citações a partir de altmetrics e comparar as métricas alternativas com a avaliação de especialistas. Desenvolvedores de aplicativos devem continuar a construir sistemas para exibir altmetrics, aplicar métodos para detectar e reparar atividade fraudulenta e criar métricas de utilização e reutilização de dados.

No futuro, uma maior participação e melhores sistemas para identificação de colaboradores especializados pode permitir que a revisão por pares seja realizada inteiramente a partir das altmetrics. Ao contrário do Fator de Impacto de periódicos, altmetrics refletem o impacto do próprio artigo, não o seu local de publicação. Finalmente, segundo Holbrook (2012), altmetrics introduzem a noção de pensar o impacto como a reutilização da pesquisa e pode oxigenar um sistema de avaliação acadêmica que muitos argumentam tornou-se excessivamente focado em métricas.

\footnotetext{
2 Processo de obtenção de serviços, ideias ou conteúdo solicitando contribuições de um grande grupo de pessoas, especialmente, a partir de uma comunidade online.
} 


\section{Cenário futuro}

O atual momento é oportuno para promover uma discussão capaz de reexaminar as métricas da ciência, que questione se a academia como uma instituição está realmente medindo aquilo que é significativo. Dados agregados de forma colaborativa podem ajudar a preencher a lacuna entre o critérios tradicionais baseados em citação e fatores de uso brutos. Taraborelli (2008) defende que modelos de avaliação distribuídos de baixo para cima (bottom-up) desafiarão métodos de avaliação mais tradicionais, em termos de cobertura, eficiência e escalabilidade. Serviços agregando indicadores de qualidade ao conteúdo científico distribuído online virá a ocupar uma função-chave no sistema de comunicação científica.

Apesar de uma crescente base empírica internacional (ALTMETRICS COLLECTION, 2012) as altmetrics baseadas em mídias sociais ainda não foram amplamente adotadas pela comunidade acadêmica brasileira. Começar a fazer uso do altmetrics para medir o impacto no contexto nacional requer tanto um conhecimento maior das propriedades e da validade destas novas métricas, como das ferramentas práticas para obtêlo. Combinadas, a exploração das mídias sociais como uma ferramenta adicional para a publicação de produtos de pesquisa e sua disseminação, é suscetível de mudar o cenário atual da comunicação científica (YEONG; ABDULLAH, 2012). Revistas de acesso aberto e sites de redes científicas estão embarcando na exploração de novas métricas para determinar o impacto da pesquisa. A comunidade acadêmica precisa movimentar estes experimentos e discussões de métricas alternativas para a fase de definições e padrões comuns, a fim de chegar a um consenso sobre o que deve ser incluído e excluído das métricas científicas.

Esses ainda são os primeiros momentos do desenvolvimento de métricas alternativas e das estruturas de apoio técnico e cultural necessários. Estamos ainda em uma fase prematura, mas se as altmetrics realmente ganharem força, concordar no início sobre seus propósitos e componentes necessários levará a adoção mais rápida por todos que precisam avaliar melhor o valor da comunicação científica.

\section{Referências}

ALTMETRICS COLLECTION. PLOS Collections. 2012. Disponível em: <http://www. ploscollections.org/altmetrics>. Acesso em: 20 dez. 2012.

BAR-ILAN, J. et al. Beyond citations: Scholars' visibility on the social Web. arXiv preprint, 25 maio 2012.2 Disponível em: <http://arxiv.org/abs/1205.5611>. Acesso em: 22 jun. 2012.

BARR, $\mathrm{K}$. The role of altmetrics and peer review in the democratization of knowledge. $2012 . \quad$ Disponível em: <http://altmetrics.org/altmetrics12/barr/>. Acesso em: 14 nov. 2012.

BIRKHOLZ, J. et al. Who are we talking about? Identifying scientific populations online. Joint Conferences of 6th China Semantic Web 
Symposium and 1st Web Science Conference. Nov, 2012. Disponível em: <http://www.researchgate.net/publication/234080016_Who_are_we_talki ng_about_identifying_scientific_populations_online/file/32bfe50eec1b4604 8f.pdf>. Acesso em: 10 jan. 2013.

CONSELHO NACIONAL DE DESENVOLVIMENTO CIENTÍ́FICO E TECNOLÓGICO (CNPq). Ajustes na Plataforma Lattes estimulam a divulgação científica. Sala de Imprensa. 14 mar. 2012. Disponível em: <http://memoria.cnpq.br/saladeimprensa/noticias/2012/0314.htm>. Acesso em: 14 mar. 2012.

CORRÊA, E. C. D. Os artigos científicos em tempos de Web 2.0: uma reflexão teórica. Revista $A C B$, v. 17, n. 1, p. 42-58, 2011.

CRONIN, B. et al. Invoked on the Web. Journal of the American Society for Information Science, v. 49, n. 14, p. 1319-1328, 1998.

CRONIN, B. Bibliometrics and beyond: some thoughts on web-based citation analysis. Journal of Information Science, v. 2, n. 1, 2001. Disponível em: <http://jis.sagepub.com/content/27/1/1.short>. Acesso em: 17 ago. 2007.

GOSLING, C. Some questions about altmetrics. 3 jan 2013. Disponível em: <http://clarileia.wordpress.com/2013/01/03/some-questions-aboutaltmetrics/>. Acesso em 10 jan 2013.

HAUSTEIN, S. Multidimensional journal evaluation: analyzing scientific periodicals beyond the impact factor. Berlim: De Gruyter/Saur, 2012. Disponível em: <http://books.google.com.br/books?id=MH1N4ottpdMC >. Acesso em: 22 nov 2012.

HAUSTEIN, S.; PETERS, I. Using social bookmarks and tags as alternative indicators of journal content description. First Monday, v. 17, n. 11.5 nov. 2012. Disponível

em: <http://firstmonday.org/htbin/cgiwrap/bin/ojs/index.php/fm/article/view/ 4110>. Acesso em: 29 nov. 2012.

HOLBROOK, B. Peer review, altmetrics, and ex ante broader impacts assessment-a proposal. ACM Web Science Conference 2012 Workshop. 2012. Disponível em: <http://altmetrics.org/altmetrics12/holbrook/>. Acesso em: 16 dez. 2012.

McFEDRIES, P. Measuring the impact of altmetrics. IEEE Spectrum, v. 49, n. 8, p. 28, ago 2012. Disponível em: <http://ieeexplore. ieee.org/stamp/stamp.jsp?tp=\&arnumber $=6247557>$. Acesso em: 29 ago. 2012.

MERTON, R. K. A crítica da ciência: sociologia e ideologia da ciência. Rio de Janeiro: Zahar, 1979.

MURAKAMI, T. Redes de citações dos periódicos com notas acima de B2 no Qualis e publicados em 2011. Bibliotecários Sem Fronteiras. 23 abril 2011. Disponível em: <http://bsf.org.br/2011/04/23/redes-de-citacoes-dos- 
periodicos-com-notas-acima-de-b2-no-qualis-e-publicados-em-2011/>. Acesso em: 23 abr 2011.

PESSONI, A. Uso da rede social Facebook como ferramenta de comunicação na área de educação em saúde: estudo exploratório produção científica da área-2005 a 2011. RECIIS, v. 6, n. 4, 2012. Disponível em: <http://www.reciis.cict.fiocruz.br/index.php/reciis/article/view/667>. Acesso em: 16 jan 2013.

PINHEIRO, L. V. R. Internet, ciência e sociedade: o que mudou para pesquisadores. ComCiência, n. 139, 10 jun 2012. Disponível em: <http://www.comciencia.br/comciencia/?section $=8 \&$ \&edicao $=79 \& i d=976>$. Acesso em: 29 ago. 2012.

PRIEM, J.; HEMMINHER, B. Scientometrics 2.0: new metrics of scholarly impact on the social Web. First Monday, v. 15, n. 7, 5 jul 2010. Disponível em:

<http://www.firstmonday.org/htbin/cgiwrap/bin/ojs/index.php/fm/article/ view/2874>. Acesso em: 18 out. 2010.

PRIEM, J. et al. Altmetrics: a manifesto. 2010. Disponível em: <http://altmetrics.org/manifesto/>. Acesso em: 14 mar. 2011.

PRIEM, J.; GROTH, P.; TARABORELLI, D. The altmetrics collection. PloS one, v. 7, n. 11, 1 nov. 2012. Disponível em: <http://www.plosone.org/article/info\%3Adoi\%2F10.1371\%2Fjournal.pone $.0048753>$. Acesso em: 12 dez. 2012.

PRIEM, J.; PIWOWAR, H.; HEMMINGER, B. Altmetrics in the wild: using social media to explore scholarly impact. arXiv preprint. 20 mar 2012. Disponível em: <http://arxiv.org/abs/1203.4745>. Acesso em: 28 mar. 2012.

PRIEM, J.; PIWOWAR, H.; HEMMINGER, B. Altmetrics in the wild: an exploratory study of impact metrics based on social media. Metrics 2011: Symposium on Informetric and Scientometric Research, New Orlean, v. 12, 2011. Disponível em: <http://jasonpriem.org/self-archived/PLoSaltmetrics-sigmetrics11-abstract.pdf>. Acesso em: 10 mar. 2012.

PRIEM, J. et al. Uncovering impacts: a case study in using altmetrics tools. Extended Semantic Web Conference Hersonissos, Grécia, 28 Mai 2012. Disponível em: <http://jasonpriem.org/self-archived/altmetrics-sepublicacameraready.pdf>. Acesso em: 22 jul. 2012.

SMEYERS, P.; BURBULES, N. How to improve your impact factor: questioning the quantification of academic quality. Journal of Philosophy of Education, v. 45, n. 1, p. 1-17, 2011.

TARABORELLI, D. Soft peer review: social software and distributed scientific evaluation. In: INTERNATIONAL CONFERENCE ON THE DESIGN 
OF COOPERATIVE SYSTEMS, 8., 2008, França. Proceedings of... França: Aix-en-Provence, 2008.2 Disponível em: <http://nitens.org/docs/spr_coop08.pdf>. Acesso em: 10 jul. 2011.

YEONG, C. H.; ABDULLAH, B. J. J. Altmetrics: the right step forward. Biomedical Imaging and Intervention Journal, v. 8, n. 3, 2012. Disponível em: <http://www.biij.org/2012/3/e15/e15.pdf>. Acesso em: $13 \mathrm{fev}$. 2013. 